tracheal irritation caused by the tube, nor was there ever any serious embarrassment in breathing.

At the necropsy, made twenty-six hours after death, on opening the thorax about three ounces of pus were found in the right pleural cavity and there was slight consolidation of the lung on this side. The trachea was healthy, with the exception of a small spot opposite the situation of the lower end of the tube, where there was a quite superficial erosion. The tongue was very short, but broad and thick; and it was noticed that, by falling back on the epiglottis, the obstruction to the passage of air would probably have been easily produced.

Remarks by Mr. HEELAS. - In this case the operation was performed for a mechanical obstruction to the breathing, which was considered to be caused by the short malformed tongue falling back on the epiglottis and thus closing the upper aperture of the larynx. By means of careful nursing the child lived for twenty-three days, during which time the general health was good up to twenty-four hours before death, when symptoms of pneumonia developed. This complication was regrettable, as there was good reason to hope that as the parts developed the condition which necessitated the operation might have passed off.

\section{NEWCASTLE-ON-TYNE ROYAL INFIRMARY.}

A CASE OF LUMBAR NEPHRECTOMY FOR CALCULOUS KIDNEY, AND ONE OF ABDOMXNAL NEPHRECTOMY FOR SARCOMA ; RECOVERY.

(Uncler the care of DR. G. H. HUME.)

The numerous cavities of the kidney in Case 1 contained each either calculi or soft calculous matter and pus. It is obvious that nephrotomy and drainage would have done nothing towards effecting a cure. The only feasible measure was nephrectomy, even though this was carried out in some uncertainty as to the working power of the other kidney. The normal state and quantity of the urine, save for the presence of pus, were evidence in favour of the latter being functionally competent. The polyuria following the operation indicates undoubtedly the strain thrown upon the remaining organ and points to increased blood pressure and some degree of vascular congestion. In Case 2 the absence of symptoms was a striking feature. Hæmaturia, which is said to occur in only about one-half the number of cases of malignant renal tumour, was probably prevented by early blocking of the ureter; and to the same cause was due the absence of other alterations of the urine. The freedom from previous exhausting loss of blood was a fortunate circumstance, influencing materially the chances of recovery. In the performance of the operation the incisions made gave ample room and rendered comparatively easy the detachment of the tumour. With regard to the nature of the growth it proved to be a large, round-celled sarcoma. It grew from the upper part of the concave border, so that unaltered kidney was expanded over the posterior aspect of the tumour. The account of these cases is taken from notes by Mr. W. Martin, house surgeon.

CASE 1. Calculous kidney; lumbar nephrectomy. - A moulder aged forty-three was admitted on Sept. 8th, 1892 complaining of pain in the back. Three years ago he had first felt this pain, which was brought on especially by movement and stooping. He had attacks, often very severe in character and lasting from a day to a month, and these attacks have been repeated down to the present time. His urine has contained a deposit of a milky colour, increasing gradually in amount. During the last twelve months his health has broken down and he has lost two stones in weight. The only important point in his previous medical history is that he was operated upon for stone in childhood. His appearance on admission was that of a feeble, anxious-looking man, who walked with stiffened gait as if in pain. He was $5 \mathrm{ft}$. $4 \mathrm{in}$. in height and weighed 6 st. $11 \mathrm{lb}$. His pulse was feeble, his temperature normal. On examining his abdomen a rounded, firm tumour about the size of a cocoanut could be felt projecting under the ribs on the left side. Below, its margin was well defined; above, the mass disappeared underneath the ribs. The swelling was dull on percussion and the dulness extended laterally and behind as high as the angle of the scapula. The mass was fixed, or moved only very slightly during forced respiration. It was tender on firm pressure. The lumbar sounds were clear. The urine was of normal quantity and composition, save that it contained a large amount of pus. During his stay in the hospital rest and treatment relieved his suffering but he steadily lost flesh at the rate of $21 \mathrm{~b}$. to $3 \mathrm{lb}$. a week.

On Sept. 26th, the presence of pus having been previously demonstrated by the hypodermic needle, the kidney was cut into by lumbar incision. A large quantity of fetid pus was evacuated, and the finger passed into the kidney found calculi and calculous matter in different cavities. It was then determined to remove the kidney and the first incision was extended towards the linea semilunaris and for a couple of inches downwards along the outer border of the rectus. In this way ample space was provided. With the finger the tumour was gradually separated from the thickened and adherent peritoneum, and as the separation went on it had to be forcibly dragged down from under cover of the ribs. The thickened pedicle, containing artery and vein, was transfixea and tied ; the dilated ureter was secured into the wound. In trimming away some of the thickened tissue in front an opening was accidentally made into the peritoneal cavity. This was closed by drawing forward the edges and tying a ligature round, instead of suturing. The operation was com. pleted by suturing the wound and placing two drainagetubes, one in the upper and the other in the lower pouch of the large cavity. During the afternoon following the operation twelve ounces of urine were passed. The evening temperature was $103^{\circ} \mathrm{F}$., the pulse 120 . The patient was not markedly collapsed. Next day the temperature fell to normal and the subsequent recovery was steady. The patient was discharged in good health at the beginning of November. During convalescence one point with regard to the quantity of urine passed was noteworthy. Before opera. tion the average daily quantity had been forty to fifty ounces: after operation there was a gradual increase, reaching ninety ounces on the sixteenth day, and up to the time of discharge the quantity continued abnormally large ; it contained a trace of albumen.

CASE 2. Sarcoma of Kidney; abdominal nephrectomy.-A clothier aged forty-one was admitted on Oct. 28th, 1892 . He stated that for a year he had been losing weight, but did not feel ill. Three months ago he first had pain in the left side, which shot across the body and down to the hip-bone, but it was not severe. A swelling in the left side was detected by his medical man a few weeks before admission. He was a thin unhealthy looking man, with acne rosacea on the face. In the abdomen a tumour, the size of a fotal head, could be felt under the left costal margin. It was nodular and hard, with an irregular outline, well defined below and passing under the ribs above. On respiration the tumour moved to the extent of an inch and a half. On percussion a broad tympanitic area was found over the front; the lumbar sounds were dull. The urine was acid, sp. gr. 1023, light in colour and contained no blood or sediment; there was no albumen or sugar.

On Oct. 31st an incision about six inches long was. made from the margin of the ribs in the linea semilunaris. The abdomen was opened and the tumour found to be in the left kidney. A lumbar incision was then made from the middle of the first cut, dividing all the structures forming the abdominal wall, including the peritoneum. The intestines. were pushed towards the right and protected with sponges. The peritoneum covering the kidney was then separatea until the whole growth was exposed. The separation was first carried out anteriorly to nearly the position of the pedicle; then, the lower end being detached, the tumour could be lifted out of its bed and drawn down. The freeing of the upper part was facilitated by a broad retractor passec under and raising the ribs. The vessels forming the pedicle were in part directly ligatured, in part clamped and afterwards tied. No bleeding of any moment took place. The large cavity left was packed with sublimate gauze dustea with iodoform, the ends of the strips being brought out through an opening made specially for drainage in the most dependent part of the loin. The whole extent of the wound was closed layer by layer with silk sutures, the skin being finally brought together with silkworm gut. Thirty-six hours after the operation the gauze was removed and a drainagetube inserted through the opening. For some days after the operation the patient had slight diarrhoea, and probabl from some fouling of the dressing, the lower part of the wound suppurated and there was a rise of temperature From the same cause it was difficult to measure the quantity 
of urine or estimate the amount of urea. At the end of a week the quantity passed in one day was forty-two ounces and contained 463.68 grains of urea. Except for the slight suppuration recovery was in all respects satisfactory, and the vatient left the hospital in good health on Nov. $29 \mathrm{th}$.

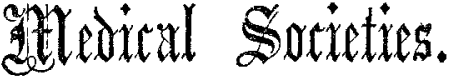

Epidemiological Sociaty.-A meeting of this Society was held on Jan. 18th, Dr. J. F. Payne, President, in the chair.-Dr. KENNWTH M LEOD read a paper on a remarkable Epidemic of Dropsy which prevailed in Calcutta in the cold season of 1877-78, 1878-79 and 1879-80 ; in Dacca, Millong and other places in Assam, Sylhet and Cachar in the cold season of 1878-79; and in the island of Mauritius from November, 1878, to June, 1879 . The facts of this singular outbreak had never been brought together systematically, and there existed differences of opinion regarding the nature, causation and identification of the disease amongst those who had studied the subject. After describing the topography and climate of Calcutta, Millong and Mauritius, and relating the incidents of the epidemic in each place, Dr. M 'Leod gave a careful summary and elaborate analysis of the symptoms as recorded by competent physicians and observed by himself. The epidemiology of the disease was then systematically discussed under the heads of : (1) The subject; (2) the environment; and (3) the origin and diffusion of the epidemic. The principal conclusions drawn from a detailed study of all the circumstances-topical, climatic, hygienic, personal and epidemiological-were: (1) That the disease was not due to coldand damp, malaria, poverty or starvation, food or water, bygienic defects, anæmia, scurvy, or any other constitutional depravity (2) that it presented the facies of an epidemic rather than of an endemic; (3) that the cause of the outbreak in Calcutta was obscure, but the probability of its conveyance from Calcutta to the other localities affected was strong; (4) that diffusion took place principally amongst persons associated in families, castes, occupation, villages \&c.; (5) that the evidence in favour of communicability was considerable, and that human agency and intercourse offered the most reasonable solution of its epidemic diffusion; (6) that the diffusibility of the disease was weak and slow, and subject to the influence of seasonal and other conditions; and (7) that the mortality was also greatly influenced by hygienic and personal conditions. After quoting the opinions recorded by Drs. Chevers, Fayrer, Moore, Davidson, Cornish, and others who had studied the disease, Dr. M'Leod contended that its symptoms and phenomena did not accord with those of Coeri-beri-a term which had evidently been applied to a variety of diseased conditions, differing in pathology and etiology, and arreeing only in the presence of dropsy and paralysis. He suggested that the disease now described should be designated simply "epidemic dropsy," and he defined it as "a specific disease characterised by the sudden appearance of anasarca, preceded in most cases by fever, vomiting, diarrhcea or irritation of the skin; often accompanied by a rash, by fever of a mild, remittent type and disorder of the bowels." The urine varies in quantity, colour and frequency, is very rarely albuminous and is never suppressed. Deep-seated pains in the limbs and dyspnoea are very common. Anæmia, which is apt to be progressive, is a prominent and constant feature of the disease. Cdema of the lungs and distension of the pleuræe and pericardium frequently occur in the later stages. The attack lasts from three to six weeks. The mortality varies from 2 to 40 per cent., according to circumstances. Death is usually caused by lung and heart complications, and is sometimes early and sudden. The disease is communicable from person to person and portable by human agency and intercourse, but its diffusion is feeble and depends on seasonal and other conditions which modify its epidemic vitality and restrict its extension and transmission.-Sir W. Moore, Dr. Manson and the President took part in the discussion. Dr. MANSON who had seen many cases of the disease, maintained that its occurrence in the cool season only, the presence of anasarca, and above all the absence of peripheral neuritis, sufficiently tistinguished it from beri-beri, and Dr. PAYNE saw no reason to doubt its malarial or telluric origin.

HARVEIAN SOCIETY OF LONDON. - A meeting of this Society was held on Jan. 5th, Mr. Bryant, Vice-President, in the
chair.-Mr. J. JACKSON CIARKE showed specimens illustrating Intestinal Obstruction-namely, Congenital Obliteration of the Small Intestine; a large Submucous Lipoma of the Ascending Colon; a Volvulus of the Sigmoid Flexure ; and a case of Extensive Ulceration of the whole of the Large Intestine.-Mr. HARRISON CRIPPs opened the discussion on Obstruction of the Large Intestine and its Treatment by Temporary Typhlotomy, He maintained that unless the obstruction can be felt in the rectum, or some definite tumour be discovered in the abdomen, its exact site in the large gut cannot be determined. In the first instance careful injections were advised, with the view to the possible washing away of an obstructing plug. If these failed to give relief, abdominal section was adrocated, Mr. Cripps recommending that in the first instance the incision should be made in the left inguinal region between the umbilicus and the anterior superior spine, and giving reasons for the selection of this spot. He gave the details of two cases in which he had found after making the abdominal incision on the left side that the sigmoid and descending colon were empty and collapsed. In each of these cases he closed the wound and opened the abdominal cavity on the right side over the cæcum. The parietal peritoneum was carefully stitched to the portion of the bowel in such a way that only a circular portion of the cæcum no bigger than a sixpenny piece was exposed. Through this an opening was made which gave immediate relief to a large quantity of air and liquid fæces. In both cases after nine and thirteen days respectively the motions commenced to pass in the natural way and in both the cæcal fistula was subsequently closed. This temporary typhlotomy was advocated in all cases where the obstruction proved to be above the descending colon, and it was moreover advised that the opening in the cæcum should in the first instance be quite small, so that it might be easily closed should the obstruction subsequently give way, and the motions pass by the right passage. Mr. GREIG-SMith preferred incision in the middle line, as it was quite possible to bring either the sigmoid or the cæcum into the wound. As time was of importance in these operations it was undesirable to make two separate openings in the abdomen. He had also performed median colotomy of the transverse colon and liked the operation very much. $\mathrm{He}$ never stitched the bowel to the skin, but preferred to stitch it to strips of sticking-plaster and to pass a skewer beneath it.-Mr. TREves spoke first of difficulties in diagnosis between obstruction in the large and small intestine. In his experience there was tenderness on pressure in impacted fæces, but not in malignant disease moreover, in freal impaction there were often symptoms of poisoning by sulphuretted hydrogen. With regard to the use of the long tube in cases of obstruction of the large bowel, he was sure that no tube could be passed beyond the sigmoid flexure. He had tried one hundred times on the dead body without success. He was inclined to agree with Mr. Greig-Smith in preferring a median opening. Mr. SHEILD thought that the administration of anæsthetics, especially ether, in cases of obstruction was fraught with danger. Should an anæsthetic be necessary he would use fresh A.C.E. mixture with plenty of air.-Dr. SIDNEx PHILLIPs said that peritonitis might extend from a case of rectal cancer and produce obstruction of the smallintestine.Mr. LockWood had seen extrusion of bowel take place through the abdominal incision; he therefore used a large number of sutures to fix the gut and close the wound in the abdominal wall. - Mr. ALLINGHAM had found the intestine insensitive to incision, whereas cutting the mesentery caused pain.-Mr. BRYANT said that ballooning of the rectum was a variable symptom, but was constant in cases of stricture. - In reply, Mr. CRIPPs said that the explanation of ballooning was very simple. In cases of stricture there was very often leakage of a small quantity of discharge into the bowel below and patients retained their flatus to avoid escape of discharge, thus blowing up the rectum. He had only met with air in the peritoneum after puncture of the intestine. $\mathrm{He}$ thought that with a median incision there would be much difficulty in bringing the cæcum or sigmoid to the skin.

SOCIETY OF MEDICAL OFFICERS OF HEALTH.-A meeting of this Society was held on Jan. 16th, Mr. Shirley Murphy, President, in the chair. A paper was read from Mr. De'Ath of Buckingham on Health Missioners in Rural Districts. The Bucks County Council had devoted a portion of the sum at their disposal for technical edncation to the support of a scheme for the diffusion of sounder knowledge of matters affecting the health of the family and the home amongst the 